\title{
OCORRÊNCIA DE CISTICERCOSE EM NECROPSIAS REALIZADAS EM UBERLÂNDIA, MINAS GERAIS, BRASIL
}

\author{
JULIA MARIA COSTA-CRUZ*, ADEMIR ROCHA *, ARNALDO M. SILVA**, \\ AZARIAS T. DE MORAES**, APARECIDA H. B. GUIMARÃES***, \\ ELIANA C. SALOMÃO $0^{* * *}$, TÂNIA M. ALCÂNTARA****
}

\begin{abstract}
RESUMO - Foram realizadas 3937 necropsias no período de 1971 a 1993 no Serviço de Anatomia Patológica do Hospital de Clínicas da Fundação de Assistência, Estudo e Pesquisa de Uberlândia, Universidade Federal de Uberlândia, Minas Gerais, local para onde convergem todos os casos de autópsias do município. A análise de 2862 registros cujos laudos estavam completos e com idade acima de um ano revelou 39 casos $(1,4 \%)$ de cisticercose. A idade variou de 16 a 83 anos, sendo $66,6 \%$ do sexo masculino. A análise da procedência demonstrou que $82,1 \%$ dos indivíduos eram do Estado de Minas Gerais e $15,4 \%$ de Goiás; 1 caso $(2,5 \%)$ não tinha procedência anotada. Dos 39 indivíduos parasitados, $35(89,7 \%)$ apresentaram comprometimento do sistema nervoso central, isolado ou associado a outras formas clínicas da doença; em 9 ocorreu a forma cardíaca (isolada ou associada), em 4 a forma muscular (isolada ou associada) e em 4 a forma visceral (isolada ou associada). Em somente 7 casos (17,9\%) a cisticercose foi a causa básica do bbito.
\end{abstract}

PALAVRAS-CHAVE: cisticercose, sistema nervoso central, necropsia.

\section{Occurrence of cysticercosis in autopsies perfomed in Uberlândia, Minas Gerais, Brazil}

SUMMARY - 3937 autopsies were performed between 1971 and 1993 in the Serviço de Anatomia Patológica of the Hospital de Clínicas of the Fundação de Assistência, Estudo e Pesquisa de Uberlândia, Universidade Federal de Uberlândia, in Minas Gerais, Brazil. At this Service of Pathology are realized all the autopsies of the municipal district of Uberlândia. The analysis of 2862 concluded autopsy reports, of death above the age of one year, disclosed 39 cases $(1.4 \%)$ of cysticercosis. The age range was 16 to 83 years and $66.6 \%$ were males; $82.1 \%$ of the patients were from Minas Gerais State, $15.4 \%$ were from Goiás State, and in one case (2.5\%) the origin was not registered. From these 39 cases, 35 (89.7\%) showed central nervous system involvement, isolated or in association to other clinical forms of the disease; in 9 occurred the isolated or associated cardiac form; in 4 the muscular form, isolated or associated, was found; 4 presented the isolated or associated visceral form. In only $7(17.9 \%)$ cases, the cysticercosis was assumed to be the direct cause of the death.

KEY WORDS: cysticercosis, central nervous system, autopsy.

O estudo da frequência e da localização do cisticerco em necropsias de indivíduos falecidos por causas diversas constitui um parâmetro para o conhecimento da epidemiologia desta zoonose. Tais dados sāo geralmente pouco divulgados, o que limita a avaliação da prevalência da parasitose

Na América Latina, estudos necroscópicos realizados no México ${ }^{1,6,13,15}$, no Chile ${ }^{4,10,22}$, na Colômbia ${ }^{14}$ e no Brasil (Tabela 1) têm contribuído para a divulgaçāo da magnitude do problema.

Departamento de Patologia da Universidade Federal de Uberlândia, Minas Gerais: * Professor Titular; ** Professor Adjunto; *** Professor Assistente; **** Professor Auxiliar. Aceite: 27 - novembro - 1994. 
O objetivo deste estudo foi analisar a ocorrência de cisticercose em necropsias do Serviço de Anatomia Patológica do Hospital de Clínicas da Fundação de Assistência, Estudo e Pesquisa de Uberlândia (SAPHCFAEPU), Departamento de Patologia, Universidade Federal de Uberlândia (DPUFU), local onde são realizadas todas as autópsias do município.

\section{MATERIAL E MÉTODOS}

Foram realizadas 3937 necropsias no SAPHCFAEPU/DPUFU, no período de 1971 a 1993 (inclusive). Foi possível a análise de 2862 registros, cujos laudos estavam completos e correspondiam a indivíduos com idade acima de um ano.

\section{RESULTADOS}

A análise dos laudos de necropsias de um período de 23 anos revelou 39 casos $(1,4 \%)$ de cisticercose em Uberlândia, Minas Gerais, sendo que em somente 7 casos (17,9\%) esta parasitose foi a causa básica do óbito. Com relação à procedência, 32 indivíduos $(82,1 \%)$ eram do Estado de Minas Gerais e $6(15,4 \%)$ de Goiás; em um $(2,5 \%)$ não foi possível a identificação.

A Figura 1 mostra a ocorrência da cisticercose ano a ano. A idade variou de 16 a 83 anos; 26 casos $(66,6 \%)$ ocorreram no sexo masculino. A Figura 2 apresenta a distribuição dos 39 indivíduos

Tabela I. Ocorrência de cisticercose em necropsias realizadas no Brasil, segundo diversos autores (1916-199J).

\begin{tabular}{|c|c|c|c|c|c|}
\hline Autor e data de publicaçāo & $\begin{array}{l}\text { Período de } \\
\text { estudo }\end{array}$ & Local & Amostragem & $\begin{array}{l}\text { No de } \\
\text { casos }\end{array}$ & $\%$ \\
\hline Almeida - $1916(2)$ & $1907-1915$ & $\begin{array}{l}\text { Hosp. Nac. } \\
\text { Alienados/RJ }\end{array}$ & 1822 & 13 & $0,7^{*}$ \\
\hline $\begin{array}{l}\text { Tretiakoff e Pacheco e Silva - } \\
1924(24)\end{array}$ & $1921-1924$ & $\begin{array}{l}\text { Hospício de } \\
\text { Juqueri/SP }\end{array}$ & 250 & 9 & $3,6^{*}$ \\
\hline Galvão - $1928(8)$ & $1916-1923$ & $\begin{array}{l}\text { Fac. Med. São } \\
\text { Paulo/SP }\end{array}$ & 997 & 15 & 1,6 \\
\hline Povoa - 1932 (19) & 1932 & Inst. Neurobiol/RJ & 1073 & 11 & $0,97 *$ \\
\hline Monteiro Salles - 1934 (16) & $1931-1934$ & $\begin{array}{l}\text { Fac.Medicina São } \\
\text { Paulo/SP }\end{array}$ & 4000 & 5 & $0,12^{*}$ \\
\hline Pinheiro e Mello - 1941 (18) & & Inst. Neurobiol. /RJ & 465 & 2 & $0,4^{*}$ \\
\hline Pupo e col. - 1945 - $1946(20)$ & $1935-1944$ & $\begin{array}{l}\text { Hospício de } \\
\text { Juqueri/SP }\end{array}$ & 1000 & 15 & $1,5^{*}$ \\
\hline Montenegro - 1946 (17) & $1923-1927$ & $\begin{array}{l}\text { Fac. Med. Cirurg. São } \\
\text { Paulo/SP }\end{array}$ & 312 & 8 & $2,5^{*}$ \\
\hline Hellmeister e Faria - 1973 (12) & $1965-1970$ & $\begin{array}{l}\text { Fac.Cien. Med. } \\
\text { Unicamp/SP }\end{array}$ & 1013 & 18 & $2,98^{*}$ \\
\hline $\begin{array}{l}\text { Guidugli Neto e Mattosinho } \\
\text { França - } 1977 \text { (21) }\end{array}$ & $1961-1974$ & $\begin{array}{l}\text { Hosp. Serv. Publ. Est. } \\
\text { São Paulo/SP }\end{array}$ & 3587 & 31 & $1,25^{*}$ \\
\hline Queiroz e Martinez - 1979 (21) & $1949-1977$ & $\begin{array}{l}\text { Hospital Edgard Stos. } \\
\text { Salvador/BA }\end{array}$ & 4000 & 13 & 0,32 \\
\hline Gobbi e col. - $1980(9)$ & $1960-1978$ & $\begin{array}{l}\text { Fac. Med. Triang. } \\
\text { Min., Uberaba/MG }\end{array}$ & 2306 & 56 & 2,4 \\
\hline Almeida e Lima Filho - 1988 (3) & $1980-1987$ & $\begin{array}{l}\text { Univ. Fed. Ceará - } \\
\text { Fortaleza/CE }\end{array}$ & 1773 & 8 & $0,5^{*}$ \\
\hline Vianna e col. - 1991 (25) & $1967-1980$ & Hosp. Sobradinho/DF & 1520 & 25 & 1,64 \\
\hline
\end{tabular}


Tabela 2. Frequência do comprometimento dos orgãos nos 39 casos de cisticercose necropsiados em Uberlânda, MG (1971-1993).

\begin{tabular}{lr}
\hline Localização & no. de casos \\
\hline \hline SNC & 48 \\
SNC + coração & 1 \\
SNC + pâncreas & 1 \\
SNC + coração + musculatura & 1 \\
esquelética & 1 \\
SNC + coraçāo + musculatura & 1 \\
esquelética + pulmão & 1 \\
Coração & 1 \\
Muscular esquelética* & 1 \\
Coração + musculatura & 39 \\
esquelética & \\
Coração + pulmão + pâncreas* & \\
\hline Total &
\end{tabular}

SNC, sistema nervoso central; *encéfalo não analisado.

Tabela 3. Localização do Cysticercus no SNC nos 35 casos de neurocisticercose.

Localização no. de casos

Córtex cerebral

Meninges

Cerebelo

Núcleos da base/tálamo 2

Ponte

1 Hemisférios cerebrais sem sede especificada

Múltiplas localizações (forma disseminada)

6

parasitados, de acordo com o sexo e a faixa etária. A Tabela 2 indica a frequência de comprometimento dos órgãos, revelando neurocisticercose em 35 casos ( $89,7 \%$ ). As localizações parasitárias no sistema nervoso central (SNC).são apresentadas na Tabela 3 . O número de Cysticercus cellulosae variou de um, em 13 casos, a vários, em 19. Em um caso, não foi registrado o número de parasitas. Somente dois casos apresentaram a variedade $C$. racemosus. $O$ acometimento cardíaco (isolado ou associado ao de outros órgãos ) foi verificado em 9 pacientes, sendo um do epicárdio, três do miocárdio e 5 disseminados. A cisticercose muscular (isolada ou associada ) foi notada em quatro pacientes, em músculos peitoral, intercostais, reto abdominal e psoas. Um dos casos de cisticercose pancreática atingia o corpo; o outro, a cauda. Dos pacientes com acometimento pulmonar, um apresentou cisticerco em lobo superior direito e outro, na pleura.

\section{COMENTÁRIOS}

A primeira referência à cisticercose humana na literatura brasileira parece dever-se ao Prof. Pedro Severiano de Magalhães, na Bahia. Ele identificou um cisticerco subcutâneo durante a dissecação de um cadáver e publicou seu achado na Gazeta Médica da Bahia em janeiro de 1881. Esse trabalho foi citado por Almeida ${ }^{2}$, que reviu a literatura nacional sobre a doença e relatou 13 casos de neurocisticercose observados em necropsias de hospital psiquiátrico do Rjo de Janeiro entre os anos de 1907 e 1915.

Desde Almeida, descreveram-se múltiplas séries de casos brasileiros de cisticercose (em geral restrita ao SNC) diagnosticada por exame necroscópico ( Tabela 1 ), com frequências variando entre $0,12 \%^{16}$ e $3,6 \%{ }^{24}$. Os dados estatísticos de 6́bitos humanos por cisticercose no Brasil, distribuídos por estados e regiōes, no período de 1980 a $1989^{5}$,demonstraram que a região Sudeste teve o maior número de casos, estando em primeiro lugar o Estado de São Paulo, seguido do Paraná e de Minas Gerais. Neste último, foram registrados 143 óbitos naquele período de 10 anos, com variação anual de 6 a 29 casos. Os números da região Centro-Oeste, embora inferiores, demonstram que Goiás apresentou, no mesmo periodo, 53 b́bitos por cisticercose. Nossos dados de 23 anos, abrangendo 0 decênio acima referido, mostram que $82,1 \%$ dos indivíduos procediam de Minas Gerais e $15,4 \%$ de Goiás. $\mathrm{O}$ acometimento de $1,4 \%$ dos indivíduos de nossa casuística insere-se dentro da faixa das observações nacionais. Gobbi et al $^{9}$, analisando os relatórios de 2306 autćpsias efetuadas em Uberaba, município do Triângulo Mineiro vizinho ao de Uberlândia, constataram 56 casos de cisticercose 


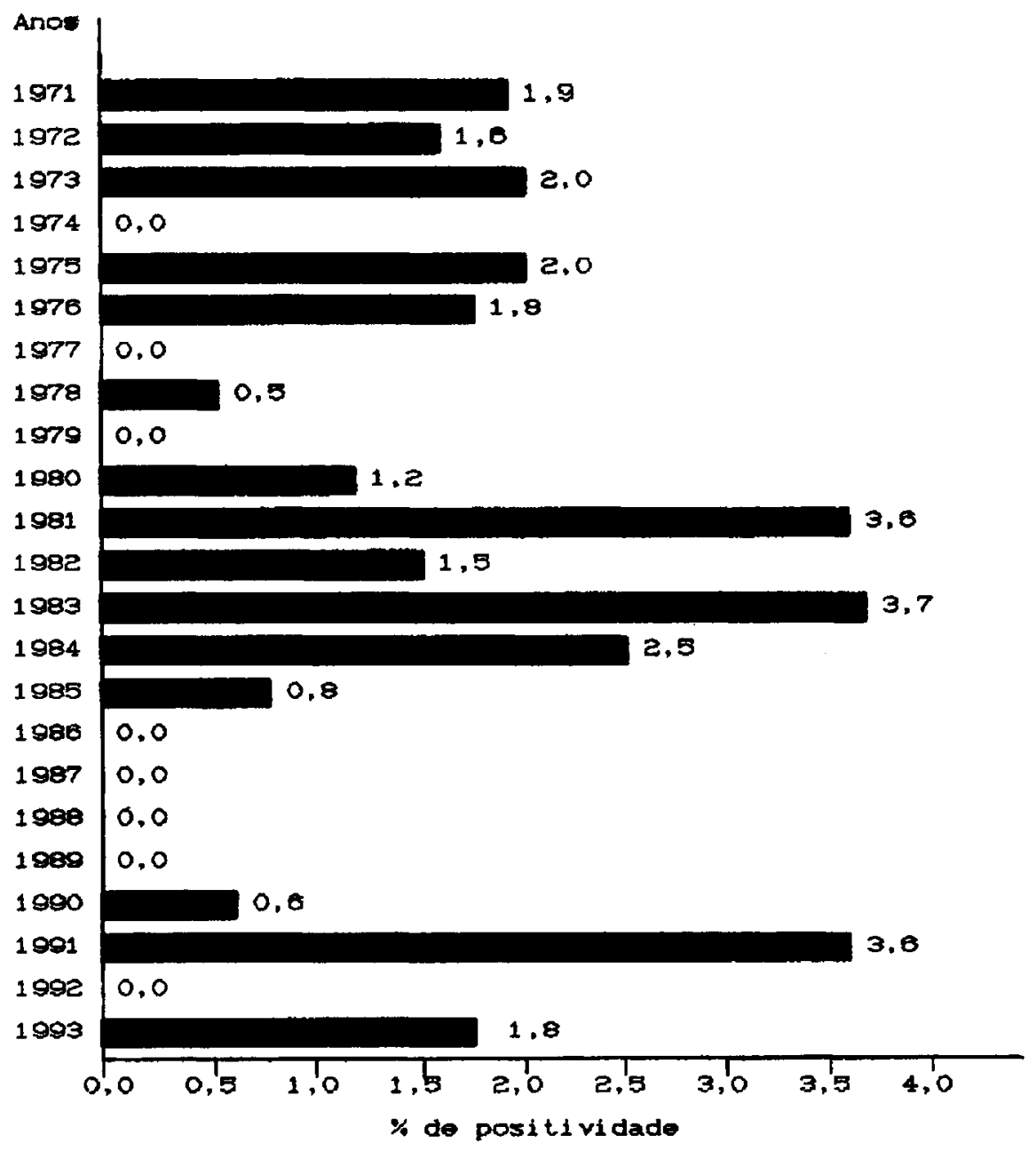

Fig 1. Ocorrencia de cisticercose em necropsias realizadas em Uberlândia, MG (1971-1993).

( $2,4 \%$ ). Seus dados, comparados aos nossos, indicam frequência significativamente maior de cisticercose naquela cidade, em relação a Uberlândia $\left(\chi^{2}=8,477\right)$. Considerando a proximidade entre os dois municípios, bem como as semelhanças entre suas populações( composição étnica, características sócio-econômicas e hábitos alimentares, entre outras), cremos que as diferentes taxas de ocorrência da parasitose se devam, basicamente, a diferenças amostrais nas populações autopsiadas.

Isolada ou como parte de doença generalizada, a cisticercose foi tida como causa básica da morte em $17,9 \%$ dos casos; este percentual quase repete o relatado por Guidugli Neto \& Mattosinho Franca $(16,1 \%)^{\prime \prime}$.

Conforme mostra a Tabela 2, o SNC é o sítio de localização mais frequente da parasitose aparecendo em 35 das nossas necropsias $(89,7 \%)$. Este fato coincide com observações prévias da literatura nacional. Em relação ao sexo e à idade, nossa casuística mostrou predomínio de neurocisticercose em homens e acima de 30 anos, confirmando a experiência de outros autores ${ }^{9.11,12}$. 


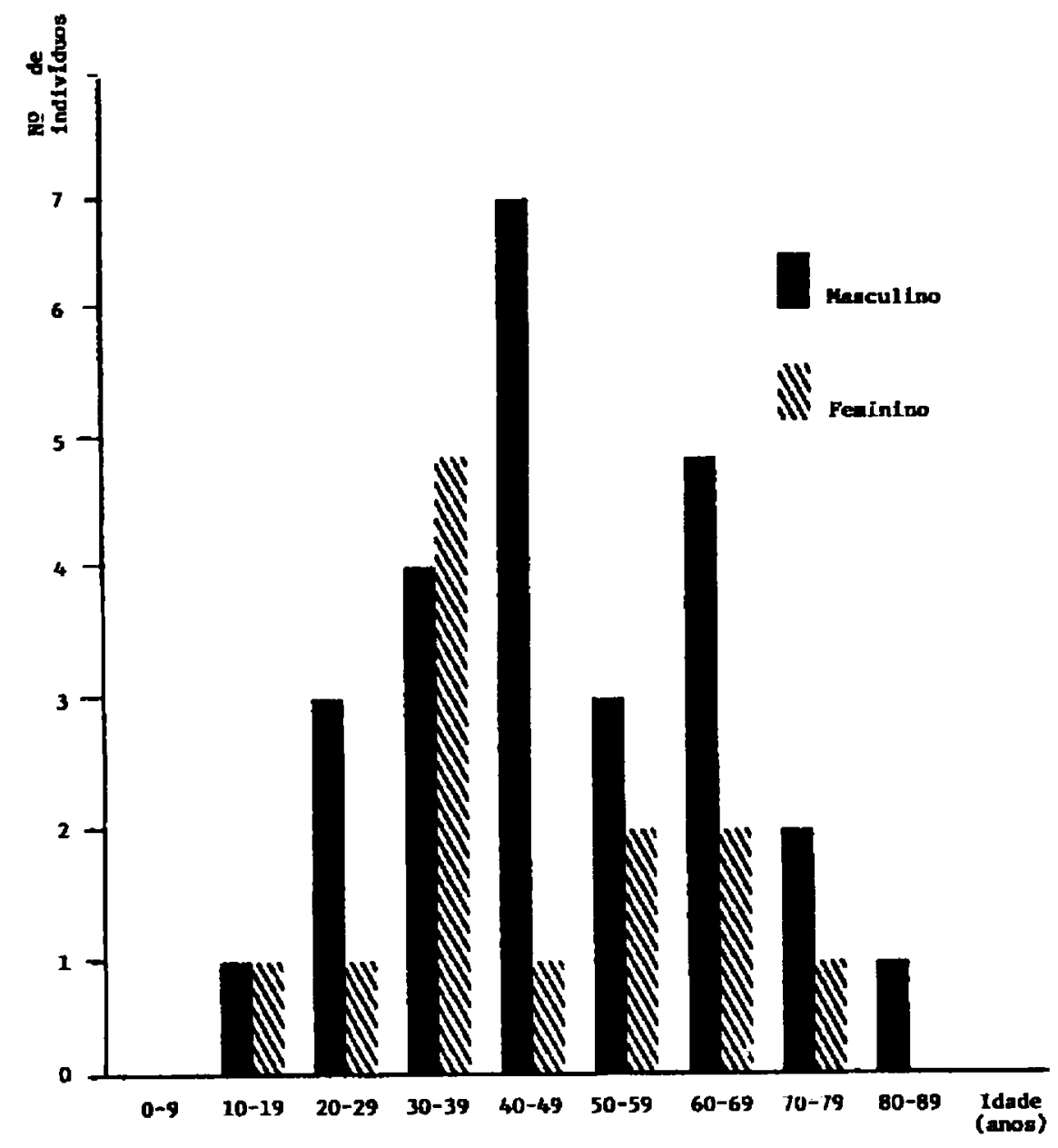

Fig 2. Distribuição dos 39 indivíduos necropsiados, com diagnóstico de cisticencose, de acordo com o sexo $e$ afaixa etária. Uberlândia, MG, 1971-1993

Nossa paciente mais jovem, portadora de neurocisticercose isolada, era uma moça de 16 anos de idade. Ferreira et al. ${ }^{7}$ observaram, em nossa região, 10 casos clínicos de neurocisticercose na infância, com variaçōes de 4 a 13 anos de idade, no período de 1983-90. A quantidade e as sedes dos cistos no SNC confirmam observações de outros autores ${ }^{11,12,21}$ A frequência da parasitose no coração (23\% dos nossos casos) superou a observada por Vianna et al. $(8 \%)^{25} \mathrm{e}$ assemelhou-se à de Gobbi et al. $(26,8 \%)^{9}$. Chamou-nos a atencão que oito dentre os nossos nove casos de cisticercose cardíaca mostraram acometimento miocárdico, isolado ou concomitante ao do epicárdio.

Nossos achados indicam que as lesões musculares, cutâneas e de outras vísceras não seriam raras na cisticercose. Observação similar foi registrada a partir de exames necroscópicos e de material cirúrgico (biópsias ) efetuados em Brasília (DF) ${ }^{25}$. É possível, no entanto, que estas formas sejam, na verdade, subdiagnosticadas, particularmente na musculatura esquelética, em decorrência da grande massa muscular do corpo e da ausência ou escassez de sintomatologia nestas sedes. A confirmar tal 
impressão, Sousa et al ${ }^{23}$, realizando tomografia computadorizada da musculatura esquelética em 8 casos com lesões do SNC, encontraram cistos intramusculares em todos.

Consideramos que o conhecimento da frequência da teníase e da cisticercose constitui um dos requisitos para planejar sua prevenção, controle e erradicação. A questão do abate clandestino de suínos é imensurável. Mesmo onde existe serviço de inspeção de carnes, sua eficiência pode ser limitada pela metodologia utilizada e pelo destino do material infectado. A melhoria no diagnóstico da cisticercose em vida (principalmente a do comprometimento cerebral) e a notificação obrigatória poderiam contribuir como alerta para a vigilância epidemiológica, motivando investigações em familiares dos pacientes e possibilitando o mapeamento das áreas endêmicas.

\section{REFERÊNCIAS}

1. Alarcón TG, Olivares LL. Cisticercosis cerebral. Rev Invest Clin 1975, 27:209.215.

2. Almeida W. Contribuição ao estudo clínico da cysticercose cerebral. Arch Bras Psiquiat Neurol Med Legal 1916, 11:229-264.

3. Almeida YM, Lima JHC Filho. Neurocisticercose no Estado do Ceará: achados de necrópsias. Rev Soc Bras Med Trop 1988, 11 (Supl): 97.

4. Barrientos J, Schirmer E, Schenone H, Aranda R, Concha L, Rojas A.Investigación de cisticercosis e hidatidosis en 5.132 autopsias practicadas en el Hospital Psiquiatrico de Santiago (1939-1966) Bol Chil Parasitol 1967, 22:150-155.

5. Brasil, Instituto Brasileiro de Geografia e Estatística. Anuário Estatístico (1980-1989). Rio de Janeiro, IBGE.

6. Briceño G, Biagi F, Martinez B. Cisticercosis: observaciones sobre 97 casos de autopsia. Prensa Med Mex 1961, 26:193-197.

7. Ferreira MS, Costa-Cruz JM., Nishioka SA, Mantese OC, Castro E, Goncalves-Pires MRF, Moura LP. Neurocysticercosis in Brazilian children: report of 10 cases. Trop Med Parasitol 1994, 45:49-50.

8. Galvão ST. Incidência e prophylaxia da cysticercose e do cysto hydatico em São Paulo. Tese. Faculdade de Medicina. São Paulo, 1928.

9. Gobbi H, Adad SJ, Neves RR, Almeida HO. Ocorrência de cisticercose (Cysticercus cellulosae) em pacientes necropsiados em Uberaba, MG. Rev Pat Trop 1980, 9:51-59.

10. Guerra F, Cortés M, Araneda T, Nunez F, Catalán J, Muñoz, R, Correa L, Schenone H. Algunas caracteristicas epidemiologicas de la hidatidosis y de la cisticercosis en la región metropolitana, Chile, 1980-1984. Bol Chil Parasitol 1985, 40:30-41.

11. Guidugli J Neto, Mattosinho-França LC. Neurocisticercose: estudo necroscópico. Rev Med IAMSPE 1977 , 8:65-67.

12. Hellmeister CR, Faria JL. Neurocisticercose: dados necroscópicos. Rev Assoc Med Bras 1973, 19:281-282.

13. Lombardo L, Mateos J, Estañol B. La cisticercosis cerebral en Mexico. Gac Med Mex 1982, 188:1-16.

14. Lopez F, Escadón A. Neurocisticercosis: estudio clinico patologico de 58 casos. Antioquia Med 1964, 14:729-743.

15. Mateos J, Biagi F, Marques H, Kretschmer R, Schnaas G. Cisticercosis como problema de salud publica. Gac Med Mex 1972, 103:225-250.

16. Monteiro Salles JM. Cisticercose cerebral. Tese, Faculdade Medicina. Sāo Paulo, 1934.

17. Montenegro J. Cegueira produzida por cisticercose cerebral. Rev Paul Med 1946, 29:348-356.

18. Pinheiro J, Mello AR. Considerações sobre a cisticercose cerebral. Arch Bras Med 1941, 31:192-212.

19. Povoa H. Cisticercose cerebral. Folha Med 1932, 13:241-246.

20. Pupo PP, Cardoso W, Reis JB, Silva CP. Sobre a cisticercose encefálica: estudo clínico, anátomo-patológico, radiológico e do líquido cefalorraqueano. Arq Assist Psicopat São Paulo 1945-1946, 10-11:3-123.

21. Queiroz AC, Martinez AMB. Envolvimento do sistema nervoso central na cisticercose. Arq Neuropsiquiatr 1979, 37:34-41.

22. Schenone $H$, Reyes $H$, Frecuencia de hidatidosis, cisticercosis y triquinosis en individuos fallecidos por muerte violenta en Santiago de Chile (1947-1966). Bol Chil Parasitol 1968, 22:62-65.

23. Sousa AQ, Diógenes IMS, Sá HLC, Costa JIF, Horta WG, Broutet N. Achados radiológicos em 44 pacientes com neurocisticercose. Rev Soc Bras Med Trop 1994, 27 (Supl 1): 331.

24. Tretiakoff C, Pacheco e Silva AC. Contribuição para o estudo da . cysticercose cerebral e em particular das lesðes cerebrais tóxicas à distância nesta afeç̧ão. Mem Hosp Juquery 1924, 1:37-66.

25. Vianna LG, Macedo V, Costa JM. Cisticercose músculo-cutânea e visceral: doença rara? Rev Inst Med Trop São Paulo 1991, 33:129-136. 